\title{
Avaliação da dor em crianças e adolescentes com disfunção cognitiva: uma revisão integrativa
}

Pain assessment in children and adolescents with cognitive dysfunction: an integrative review

Evaluación del dolor en niños y adolescentes con disfunción cognitiva: una revisión integradora

Stela Faccioli Ederli ${ }^{1}$ (D) https://orcid.org/0000-0001-8021-0750

Mauren Teresa Grubisich Mendes Tacla ${ }^{2}$ (D) https://orcid.org/0000-0001-8928-3366

Sara Lima Aires Braz ${ }^{1}$ (D) https://orcid.org/0000-0003-1411-3359

Thalita Gabriela Silva Rosseti ${ }^{1}$ (D) https://orcid.org/0000-0002-4030-9630

\section{Resumo}

Objetivo: Identificar na literatura evidências sobre a avaliação da dor em crianças e adolescentes com disfunção cognitiva.

Métodos: Revisão integrativa da literatura, realizada nas bases de dados PubMed, MEDLINE e LILACS em fevereiro de 2019, utilizando os critérios de inclusão: resumos e/ou títulos que contemplassem a dor em crianças e/ou adolescentes com disfunção cognitiva; estudos publicados nos últimos 10 anos nos idiomas inglês, português e espanhol. Foram excluídos da amostra os estudos duplicados e os provenientes de literatura cinzenta.

Resultados: A amostra final foi composta de 24 artigos e os resultados foram classificados em três categorias: Características da dor em crianças com disfunção cognitiva; Avaliação da dor e instrumentos validados; Papel do enfermeiro no contexto da avaliação da dor.

Conclusão: Evidenciou-se a importância da utilização de instrumentos validados para a avaliação adequada da dor nessa população.

\section{Abstract}

Objective: Identify in the literature evidences about the evaluation of pain in children and adolescents with cognitive dysfunction.

Methods: Integrative literature review, performed in the databases PubMed, MEDLINE and LILACS in February 2019, using the following criteria of inclusion: abstracts or titles that contemplated pain in children or adolescents with cognitive dysfunction; studies published over the last 10 years in English, Portuguese and Spanish. Duplicated studies and studies originated from grey literature were excluded from the sample.

Results: The final sample was composed by 24 articles and the results were classified under three categories: Characteristics of pain in children with cognitive dysfunction; Evaluation of pain and validated instruments; Role of the nurse in the context of pain evaluation.

Conclusion: It became evident the importance of the utilization of validated instruments for an adequate evaluation of pain in this population.

\section{Resumen}

Objetivo: Identificar en la literatura evidencias acerca de la evaluación del dolor en niños y adolescentes con disfunción cognitiva.

Métodos: Revisión integradora de la literatura, ejecutada en las bases de datos PubMed, MEDLINE y LILACS en febrero de 2019, utilizando los siguientes criterios de inclusión: resúmenes o títulos que contemplaran el dolor en niños o adolescentes con disfunción cognitiva; estudios publicados en los últimos 10 años en los idiomas inglés, portugués y español. Fueran excluidos de la muestra los estudios duplicados y estudios provenientes de literatura gris.

Resultados: La muestra final fue compuesta por 24 artículos y los resultados fueran clasificados en tres categorías: Características del dolor en niños con disfunción cognitiva; Evaluación del dolor e instrumentos validados; Papel del enfermero en el contexto de la evaluación del dolor.

Conclusión: Quedó evidente la importancia de la utilización de instrumentos validados para la evaluación adecuada del dolor en esa población.

\section{Descritores}

Medição da dor; Disfunção cognitiva; Enfermagem pediátrica; Criança

\section{Keywords}

Pain measurement; Cognitive dysfunction; Pediatric nursing; Child

\section{Descriptores}

Dimensión del dolor; Disfunción cognitiva; Enfermería pediátrica; Niño

\section{Como citar:}

Ederli SF, Tacla MT, Braz SL, Rosseti TG. [Pain assessment in children and adolescents with cognitive dysfunction: an integrative review]. Rev Soc Bras Enferm Ped. 2020;20(1):47-53. Portuguese 


\section{Introdução}

A dor é um fenômeno universal, que acarreta sofrimento humano e compromete a qualidade de vida de inúmeros indivíduos. Alguns países, como os EUA, declararam a primeira década do século XXI como a Década de Controle e Pesquisa da Dor. E, atualmente, a International Association for the Study of Pain (IASP) declarou o ano de 2019 como o Ano Global Contra a Dor em Grupos Vulneráveis. ${ }^{(1)}$

Dentre os grupos vulneráveis, encontra-se o de crianças com déficit intelectual e cognitivo associados às deficiências neurológicas. Porém, ainda que essa população receba a classificação de vulnerabilidade pela IASP, é escassa a produção científica quanto à avaliação e manejo da dor nessas crianças. ${ }^{(2)}$

O termo deficiência no desenvolvimento inclui uma população de crianças que é altamente heterogênea: crianças com agravos de saúde de tipo e etiologia diversos, como a síndrome de Down, a paralisia cerebral (PC), a síndrome de Rett, além da inclusão de crianças com deficiências no desenvolvimento neurológico de origem desconhecida. Portanto, pela própria natureza de sua condição subjacente, essas crianças estão particularmente em risco para experiências dolorosas desproporcionais aos seus pares com desenvolvimento adequado para suas faixas etárias. ${ }^{(3)}$

No entanto, não há razões para acreditar que a dor seja menos frequente em uma criança com deficiência no desenvolvimento, ou que esse indivíduo seja insensível ou indiferente à mesma. Inúmeras limitações funcionais, bem como a própria condição neurológica subjacente, frequentemente confundem a manifestação do quadro doloroso, independentemente do grau de incapacidade. Assim sendo, a dor costuma fazer parte da vida cotidiana desses indivíduos. ${ }^{(4)}$

Baseado nisso e nas inconsistências na sua aplicação, a definição de dor da IASP que refere-se a "uma experiência sensorial e emocional desagradável associada a danos teciduais reais ou potenciais, ou descrita em termos de tais danos" ${ }^{\prime 5}$ foi revisada em 2002, em parte, com o requisito implícito de relatar a experiência sensorial ou emocional, com a cláusula 'a incapacidade de se comunicar verbalmente não nega a possibilidade de um indivíduo sentir dor e precisa de tratamento adequado para aliviá-la'.(6)
A abordagem majoritária de avaliação da dor, no entanto, ainda depende extensivamente do autorrelato, usando a linguagem baseada na palavra falada. ${ }^{(7)}$ Contudo, as crianças e adolescentes com disfunção cognitiva não conseguem praticar o autorrelato por terem deficiências neurológicas ou de comunicação. O principal meio de avaliação da dor consiste na observação do comportamento, que é complementada com o conhecimento do contexto e informações partilhadas pelos pais e sinais fisiológicos da dor. ${ }^{(1)}$

Ao prestar assistência a dor às crianças e adolescentes com deficiência cognitiva, os profissionais de saúde podem adivinhar ou fazer suposições com base em 'pistas', como padrões de comportamentos observados como forma de comunicação. No entanto, existem vários níveis de funcionamento cognitivo, habilidades verbais, deficiências físicas e motoras, de modo que nenhuma avaliação se encaixa em todas as crianças com disfunção cognitiva. Além disso, muitas vezes, há várias pessoas envolvidas no cuidado das mesmas e, portanto, a avaliação e o manejo da dor podem ser dificultados. ${ }^{(8)}$

Diante dos pressupostos acima, o presente estudo justifica-se pela escassez de pesquisas relacionadas às características e à avaliação da dor em crianças e adolescentes com disfunção cognitiva, tendo em vista que em muitas situações a dor é avaliada de maneira não fidedigna. A contento, este trabalho tem como objetivo identificar na literatura aspectos relacionados à avaliação da dor e ao papel do enfermeiro nesse contexto.

Considerando a importância do estudo, os resultados contribuirão com a qualidade da assistência multiprofissional na avaliação da dor, tendo em vista que as intervenções realizadas de maneira sistematizadas e fidedignas são fundamentais para o tratamento proposto e avaliação da terapêutica aplicada.

\section{Métodos}

O presente estudo constitui-se como uma revisão integrativa da literatura baseado em cinco estágios, sendo eles: a identificação do problema; as estratégias de busca na literatura; a avaliação dos dados focada na qualidade e representatividade dos dados; a análise dos dados que inclui a exibição, comparação e conclusões; e, por fim, a apresentação, que sintetiza as descobertas e descreve as implicações para a prática, política e pesquisa. ${ }^{(9)}$ 
A primeira etapa da revisão foi conduzida com a utilização da estratégia PICO (Patient, Intervention, Control, Outcome) adaptada para pesquisa não clínica, sendo utilizados População, Interesse e Contexto. As características da população selecionada devem ser baseadas em uma justificativa clara, já que a amostra deve assegurar a representatividade dela no fenômeno em análise. $\mathrm{O}$ fenômeno de interesse corresponde ao nível de detalhe atribuído e pode variar com a natureza ou complexidade do tema. Por fim, o contexto poderá variar de acordo com o objetivo da revisão e das perguntas específicas construídas para atingir o objetivo. Desse modo, foi considerado P (crianças e adolescentes), C (disfunção cognitiva) e I (Avaliação da dor).

Frente a essa etapa, definiu-se a seguinte pergunta norteadora para conduzir o desenvolvimento da pesquisa: "Como é realizada a avaliação da dor em crianças e adolescentes com déficits cognitivos?".

Para a segunda etapa, não foram empregados descritores não controlados, mas foram utilizados os seguintes descritores controlados combinados com operadores booleanos dispostos no Medical Subject Headings (MeSH) e Descritores em Ciências da Saúde (DECs): Pain, Cognitive Dysfunction cognitive impairment, Pain Measurement, Pediatric nursing. Os descritores foram combinados de diferentes formas para garantir uma busca ampla e não foi determinado um campo específico de busca, mas optou-se por all fields.

$\mathrm{Na}$ terceira etapa, caracterizada como coleta de dados, foram incluídas na pesquisa as publicações indexadas às seguintes bases de dados: US National Library of Medicine (PubMed), Biblioteca Virtual em Saúde (MEDLINE) e Latin American And Caribbean Health Sciences Literature (LILACS). A busca foi realizada em fevereiro de 2019.

A seleção dos estudos baseou-se nos seguintes critérios de inclusão: resumos e/ou títulos que contemplassem a dor em crianças e/ou adolescentes com déficit cognitivo, estudos primários e revisões publicados nos últimos 10 anos nos idiomas inglês, português e espanhol. O período de 10 anos para seleção dos estudos estabeleceu-se considerando a maior disseminação dos estudos a partir de 2009. Foram adotados como critérios de exclusão os estudos duplicados e os provenientes de literatura cinzenta, classificados como publicações não-convencionais, assim como a exclusão dos editoriais, teses e dissertações.
Na quarta etapa de análise, os estudos foram avaliados de maneira rigorosa e a busca nas bases de dados com o cruzamento dos descritores. Sendo assim, a pesquisa resultou em 115 artigos e, após serem aplicados os critérios de inclusão e exclusão, totalizaram 44 para leitura na íntegra. Destes, foi selecionado um total de 24 artigos, que contemplavam o objetivo da revisão, como apresentado no fluxograma (Figura 1).

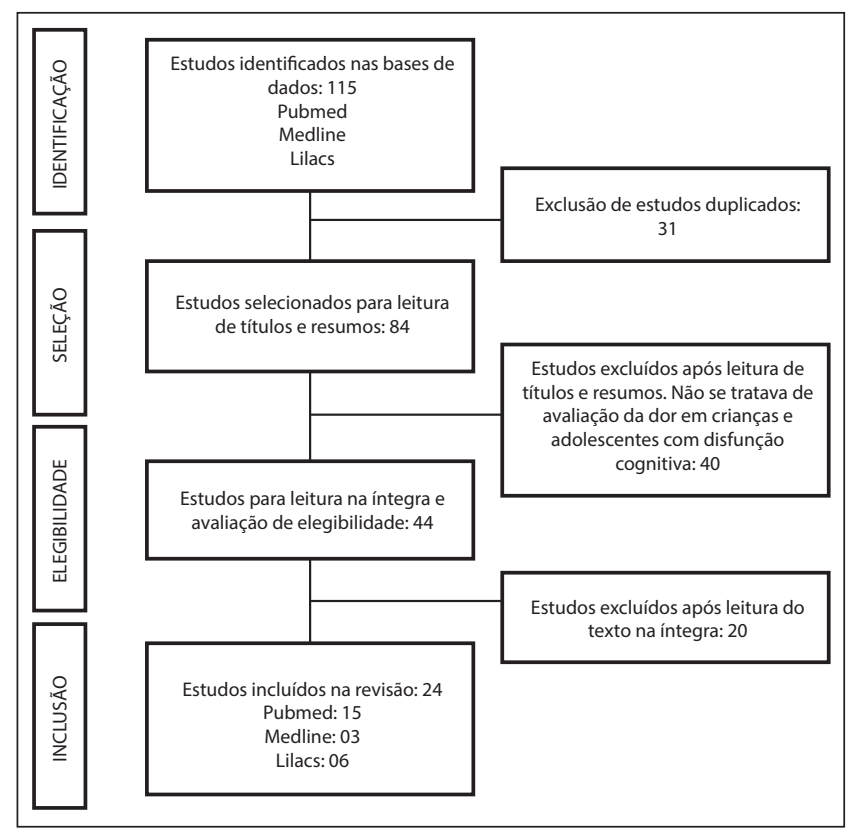

Figura 1. Fluxograma de identificação e seleção dos estudos

Os artigos foram avaliados por dois pesquisadores de forma independente considerando os critérios de inclusão e exclusão e norteados pela pergunta de pesquisa. Cada pesquisador registrou as informações extraídas das buscas e, após as comparações, a discordância foi solucionada por meio de consenso entre os pesquisadores. Por fim, na sexta etapa realizou-se a interpretação dos resultados.

A análise culminou com a criação de três categorias a serem apresentadas: Características da dor em crianças com déficit cognitivo; Avaliação da dor e instrumentos validados; Papel do enfermeiro no contexto da avaliação da dor.

\section{Resultados}

Dentre as 24 publicações, 11 (46\%) foram publicadas entre 2017 e 2019, sete (29\%) entre os anos de 2013 a 
2016 e seis (25\%) entre os anos de 2009 a 2012. Em relação aos tipos de estudos incluídos, seis (25\%) eram estudos de método misto, cinco (21\%) estudos descritivos, quatro $(16 \%)$ revisões integrativas, quatro $(16 \%)$ estudos metodológicos, três (13\%) estudos exploratórios e duas (8\%) revisões sistemáticas.

Em relação à origem dos estudos, dois foram realizados na Itália (8\%), seis no Canadá (25\%), 12 foram desenvolvidos nos Estados Unidos (50\%), e nos países Brasil, Irlanda, Alemanha e Inglaterra foram realizados um estudo em cada um deles (1\%).

No quadro 1, encontra-se a síntese dos principais aspectos apresentados nos textos segundo a categoria temática.

Quadro 1. Distribuição das categorias temáticas na análise dos artigos, de acordo com os principais aspectos apresentados

\begin{tabular}{|c|c|}
\hline Categorias Temáticas & Principais aspectos apresentados \\
\hline $\begin{array}{l}\text { Características da } \\
\text { dor em crianças com } \\
\text { déficit cognitivo }\end{array}$ & $\begin{array}{l}\text {-Conceito e influências no processo doloroso. }{ }^{(10-12)} \\
\text {-Condições e fatores predisponentes. }{ }^{(13,14)} \\
\text {-Comportamentos e particularidades diante da } \\
\text { dor }{ }^{(2,8,15-18)}\end{array}$ \\
\hline $\begin{array}{l}\text { Avaliação da dor e } \\
\text { instrumentos validados }\end{array}$ & $\begin{array}{l}\text {-Interpretação da dor por parte dos profissionais. }{ }^{(19,20)} \\
\text {-Papel dos pais e responsáveis na avaliação. }{ }^{(15)} \\
\text {-Avaliação subjetiva da dor. }{ }^{(10,21,22)} \\
\text {-Componentes dos instrumentos de } \\
\text { avaliação. }{ }^{(2,8,11,12,14,18,23-25)} \\
\text {-Escassez de instrumentos validados.(8) }\end{array}$ \\
\hline $\begin{array}{l}\text { Papel do enfermeiro } \\
\text { no contexto da } \\
\text { avaliação da dor }\end{array}$ & $\begin{array}{l}\text {-Cuidado multidisciplinar baseado em evidências. }{ }^{(13)} \\
\text {-Avaliação fidedigna e sistematizada da dor como } \\
\text { quinto sinal vital. }{ }^{(10,18,26)} \\
\text {-Manejo da dor incluso no processo de } \\
\text { enfermagem. }^{(8,27)} \\
\text {-Acolhimento e escuta qualificada.(28) }\end{array}$ \\
\hline
\end{tabular}

\section{Discussão}

A análise dos estudos selecionados possibilitou a sintetização em três categorias temáticas que contemplam as características da dor em crianças e adolescentes com déficit cognitivo, a avaliação da dor e instrumentos validados e o papel do enfermeiro no contexto da avaliação da dor.

\section{Categoria temática 1 - Características da dor em crianças com déficit cognitivo}

A dor, por ser considerada como o quinto sinal vital, deve ser avaliada de maneira sistemática, assim como os demais sinais vitais e é necessário considerar as alterações em sua intensidade e caráter, podendo ser classificada de três formas: leve, moderada e grave. É uma experiência sensorial e emocional muito desagradável e influenciada por diversos fatores. Dentre esses pelas experiências anteriores frente à dor, pela própria cultura e as redes de apoio social. ${ }^{(10-12)}$

As crianças com déficit cognitivo estão mais vulneráveis à dor, pois apresentam problemas clínicos que podem causar o fenômeno, além da fisiologia relacionada à lesão neurológica. Essas crianças são continuamente expostas a procedimentos dolorosos e pode-se dizer que exibem características comportamentais peculiares, que podem ocultar a expressão de dor. Estudos indicam aumento da probabilidade da presença de dor em crianças com déficit cognitivo e ausência de comunicação, sendo, portanto, de extrema importância que se tenha um manejo apropriado dessa população. ${ }^{(13,14)}$

Crianças em crise de dor podem apresentar-se irritáveis, infelizes e inconsoláveis, mas também podem estar quietos e não ativos. Estudo realizado nos Estados Unidos com 46 crianças com déficit cognitivo indicou que $75 \%$ sofriam de dor ao menos uma vez ao dia e $88 \%$ dessas crianças sentiam dor semanalmente. ${ }^{(21)}$

Comportamentos específicos de dor em crianças com déficit cognitivo foram identificados por meio de estudos, de observações e de questionamentos direcionados aos pais e/ou cuidadores, que notaram tais comportamentos quando esses menores estão com dores. Os comportamentos encontrados foram: choro, gemidos ofegantes, ingestão aguda de ar, caretas, testa e olhos franzidos, olhos bem abertos, dentes cerrados, dentes rangendo, olhar angustiado, incapacidade de ser consolada, busca por conforto, aumento ou diminuição do sono, aumento dos movimentos dos braços e pernas, voltas ou reviravoltas, enrijecimento das extremidades, apertos de punhos, arqueamento das costas, taquicardia, sudorese, tremores, mudança de cor da pele, lágrimas, expressão facial embotada e, também, comportamentos de dor atípicos, como rir. ${ }^{(2,15)}$ Ainda, nestes estudos é relatado que essas dores podem apresentar duração de até nove horas. ${ }^{(2,15,16)}$

Segundo um estudo canadense, ${ }^{(8)}$ cerca de $45 \%$ dos pais de crianças com déficit cognitivo severo a profundo relataram que seu filho apresentou comportamentos indiretos quando estava sofrendo com dores, sendo $22 \%$ com choro e $10 \%$ com modificações no comportamento. Esses foram os sinais mais comuns. Estudos 
evidenciam que crianças mais velhas apresentam mais episódios dolorosos do que as mais jovens. ${ }^{(17,18)}$

\section{Categoria temática 2 - Avaliação da dor e instrumentos validados}

O tratamento da dor em crianças com déficit cognitivo está relacionado à grande dificuldade em interpretar os comportamentos de dor nesse público, devido aos distúrbios motores ou sensoriais e à ausência de ferramentas/instrumentos de avaliação fidedignas e válidas para essa população. Quando esta não pode relatar a sua dor, os profissionais interpretam os comportamentos fundamentando-se em crenças, experiências e ações pessoais. ${ }^{(20)}$

Esses instrumentos envolvem os pais, cuidadores e enfermeiros para que determinem as características que ocorrem com as crianças desde o início do fenômeno doloroso. Os pais relatam que acham a identificação da dor uma operação incerta, mas que conseguiriam constatar com rapidez os comportamentos de dor nos seus filhos caso possuíssem em mãos a ferramenta de avaliação. Vale ressaltar que, além do uso das ferramentas, deve-se obter outras informações como duração e gravidade dos episódios de dor, para que possam discernir se essas informações diminuíram positivamente após as intervenções. ${ }^{(15)}$

As crianças com deficiência cognitiva moderada e grave não têm capacidade suficiente para conseguirem expor sua dor e cerca de $75 \%$ das crianças com deficiência cognitiva leve não conseguem explicar a sua dor e a intensidade. ${ }^{(10)}$ Mesmo assim, é observado na prática que enfermeiros e cuidadores aplicam escalas de auto relato para crianças com necessidades especiais. Estudos que compararam as avaliações dos enfermeiros identificaram que esses profissionais subestimam a intensidade da dor das crianças, ou seja, têm utilizado sua própria experiência e intuição para avaliar a criança, o que não traduz os resultados de avaliação dos instrumentos. . $^{(10,21,22)}$

Existem instrumentos validados para a avaliação da dor de crianças e adolescentes com déficit cognitivo, entre eles temos o Non-Communicating Children's Pain Checklist-Postoperative Version (NCCPC-PV). Esse instrumento é o mais citado na literatura voltado para essas crianças e adolescentes que não se comunicam e estão na faixa etária entre 3 a 18 anos. É composto por
27 itens, através dos quais os observadores estabelecem uma pontuação para cinco eixos que ao final serão somados. Escore menor que 11 significa ausência de dor ou dor leve, escore maior que 11 indica que a criança está com dor moderada a grave. A escala exige que o profissional ou cuidador faça uma observação por um período de 10 minutos antes de definir o escore. ${ }^{(8)}$

Há também outra versão deste instrumento, o Non-Communicating Children's Pain Checklist - Revised (NCCPC-R), desenvolvido nos anos de 1990. Trata-se de uma variante revisada, composta por 30 itens que contemplam os comportamentos das áreas vocal, social, facial, atividade, corpo e membros, parte fisiológica, comer e dormir. Foi testado com crianças não comunicativas verbalmente por um período de 2 horas, sendo que escores acima de sete indicam presença de dor. Pode ser utilizado por qualquer indivíduo que esteja relacionado ao cuidado com a criança. ${ }^{(2,11,12,14,18,22,23)}$ Não foram encontrados estudos que traduziram e validaram o instrumento para a língua portuguesa falada no Brasil.

O instrumento Paediatric Pain Profile (PPP) é uma escala de classificação comportamental para avaliar a dor em crianças com graves comprometimentos físicos e de aprendizagem, e foi desenvolvido por pesquisadores do Royal College of Nursing Institute, em Oxford, e do Institute of Child Health, em Londres. Ele aborda o histórico de dor da criança, avaliação inicial e contínua das dores, intervenções, resultados e a discussão com os profissionais em relação à dor da criança. No PPP são incluídos 20 itens classificados em uma escala Likert de quatro pontos. Uma pontuação maior que 14 indica dor moderada a grave. Esse instrumento avalia a dor pelos movimentos e reações, sendo necessário observar por cinco minutos antes de definir a pontuação. $(2,12,21,22,28)$

Além dos instrumentos já citados, existem outras ferramentas de avaliação de dor para crianças com deficiência, como, por exemplo, a Pain and Discomfort Scale (PADS) que possui 18 itens de avaliação. Esse instrumento é baseado nas expressões faciais, do corpo e de movimentos que possam indicar dor aguda e desconforto. ${ }^{(8,14)}$ Há também o Disability Distress Assessment Tool - (DIS DAT), que auxilia na observação e identificação de sinais de angústia em pessoas que têm comunicação severamente limitada por conta de comprometimento cognitivo e consiste em cinco itens que 
avaliam os comportamentos da face, expressão facial, posição/movimento da perna, atividade, choro/ vocal, consolabilidade. ${ }^{(8,14)}$

Segundo a Nursing Assessment of Pain Intensity (NAPI), a dor também pode ser avaliada pelos parâmetros fisiológicos, como a respiração, coloração da pele, sudorese, urina, sangue, pressão arterial e frequência cardíaca, além das mudanças no comportamento. Estudiosos afirmam que os parâmetros fisiológicos são bem vistos quando a dor é aguda, pois na dor crônica o corpo se adapta e pode não demonstrar essas alterações fisiológicas. ${ }^{(19,26)}$

Pesquisa realizada com crianças com déficit cognitivo utilizou o Pediatric Pain Coping Inventory (PPCI), que inclui perguntas para os responsáveis sobre a criança em relação ao que ela pensa ou faz quando está com dor e, com que frequência, essas estratégias são utilizadas. Esse material auxilia os pais/responsáveis a conhecerem seus filhos e saber identificar quando eles apresentam dor. ${ }^{(18)}$

Por fim, temos a Individualized Numeric Rating Scale (INRS), que consiste na adaptação da escala de classificação numérica, que define a intensidade da dor das crianças por meio dos relatos dos pais. Os profissionais entrevistam os pais ou responsáveis pela criança para descreverem os comportamentos de dor, cuja classificação varia de zero a 10, sendo zero = sem dor, cinco $=$ dor moderada e 10 = pior dor possível. Esse instrumento baseia-se unicamente nos indicadores individuais de dor da criança e também pode ser utilizado por enfermeiros em ambientes hospitalares. ${ }^{(2,25)}$

Embora sejam instrumentos extremamente importantes, não foram encontradas na literatura versões traduzidas para o idioma português. Há, pois, a necessidade de validação para outras línguas além das originais, tendo em vista que muitos países não as utilizam por falta de adaptação cultural e semântica. ${ }^{(8)}$

\section{Categoria temática 3 - Papel do enfermeiro no contexto da avaliação da dor}

Devido à grande complexidade que envolve a assistência a essas crianças e suas famílias, a avaliação da dor não deve focar somente no âmbito da avaliação física das crianças, mas também enfocar o contexto social, espiritual e psicológico. Então, a enfermagem precisa adotar práticas baseadas em evidências e fundamentar-se na multidimensionalidade da dor, ressaltando o trabalho multidisciplinar para o manejo adequado da dor. ${ }^{(13)}$

Os enfermeiros necessitam identificar e utilizar instrumentos fidedignos para avaliar a dor das crianças e devem dominar essas ferramentas, além de ensinar novas abordagens no enfrentamento da dor para esse público e familiares, como, por exemplo, as estratégias de autoinstrução cognitiva e distração que ainda são pouco utilizadas. É importante que esses profissionais identifiquem meios de avaliação da dor válidos para que os pais e a equipe possam padronizar a sua avaliação, para que , assim, seja garantido que eles avaliem a dor utilizando seu conhecimento e considerando que existem diversas maneiras pelas quais as crianças transmitem a sua dor. ${ }^{(10,18,26)}$

Para a American Nurses Association (ANA), o papel do enfermeiro frente a dor inclui a avaliação, planos para estratégias farmacológicas para o controle da dor, programação e avaliação da resposta do paciente às intervenções, ou seja, inclui todo o processo de enfermagem, visto que a avaliação observacional da dor, geralmente realizada por enfermeiros, é fundamental para o manejo adequado da dor de crianças com déficit cognitivo. ${ }^{(8,27)}$

Destarte, os enfermeiros devem praticar uma escuta qualificada com os pais ou responsáveis pelas crianças, tendo em vista que possuem muito conhecimento sobre seus filhos, sendo essas informações de extrema importância na tomada de decisão e no manejo da dor pelo profissional. ${ }^{(28,29)}$

\section{Considerações finais}

Por meio dessa revisão de literatura, foi possível identificar, perante a complexidade e multidimensionalidade do fenômeno doloroso, as particularidades da criança e do adolescente com disfunção cognitiva, que dificulta e até impossibilita a avaliação adequada da dor. Além disso, foi possível comprovar a importância e condição sine qua non da utilização de instrumentos adaptados e validados para a avaliação da dor dessa população. Evidenciou-se que a equipe de enfermagem possui papel fundamental na avaliação da dor, porém ainda há profissionais e pais/responsáveis que 
desconhecem os instrumentos e avaliam a dor de maneira subjetiva, expondo a criança a períodos de dor sem manejo adequado. Como implicação para a enfermagem, pode-se reafirmar a importância da equipe de enfermagem na avaliação adequada da dor como sinal vital e do enfermeiro como condutor do manejo adequado. Por fim, faz-se necessário refletir sobre a escassez de publicações da temática abordada nessa revisão, principalmente de estudos metodológicos que poderiam validar os instrumentos aqui apresentados para a língua portuguesa falada no Brasil.

\section{Referências}

1. Lewis VT. The ongoing quandaries of behavioral pain assessment in children with neurocognitive impairment. Dev Med Child Neurol. 2011;53(2):106-7.

2. Crosta QR, Ward TM, Walker AJ, Peters LM. A review of pain measures for hospitalized children with cognitive impairment. J Spec Pediatr Nurs. 2014;19(2):109-18.

3. Brandow AM, Hansen K, Nugent M, Pan A, Panepinto JA, Stucky CL. Children and adolescents with sickle cell disease have worse cold and mechanical hypersensitivity during acute painful events. Pain. 2019;160(2):407-16.

4. Oberlander TF, Symons FJ, Burkitt CC. Pain in individuals with intellectual disabilities: a practical guide. $1^{\text {a }}$ ed. Wiley-Blackwell: 0xford; 2010.

5. Merskey H, Bogduk N. Classification of Chronic Pain. IASP Pain Terminology. International Association for the Study of Pain. PAIN. 2012.

6. Solé E, Galán S, Vega R, Castarlenas E, Sánchez-Rodríguez E, Jensen MJ, et al. Psychometric properties of the Functional Disability Inventory for assessing Pain-related disability in children from the community. Disabil Rehabil. 2019;41(20):2451-8.

7. Breau LM, Burkitt C.Assessing pain in children with intellectual disabilities. Pain Res Manag. 2009;14(2):116-20.

8. Hopia H, Latvala E, Liimatainen L. Reviewing the methodology of na integrative review. Scand. J. Caring Sci. 2016;30(4):662-9.

9. Zisk-Rony RY, Lev J, Haviv H. Nurses' report of in-hospital pediatric pain assessment: examining challenges and perspectives. Pain Manag Nurs. 2015;16(2):112-20.

10. Pascolo P, Peri F, Montico M, Funaro M, Parrino R, Vanadia F, et al. Needle-related pain and distress management during needle-related procedures in children with and without intellectual disability. Eur J Pediatr. 2018;177(12):1753-60.

11. Brand K,Al-Rais A. Pain assessment in children. Paediatr Anaesth. 2019;17(6):270-3.
12. Bussotti EA, Pedreira ML. Dor em crianças com paralisia cerebral e implicações na prática e pesquisa em enfermagem: revisão integrativa. Rev Dor. 2013;14(2):142-6.

13. Doody 0, Bailey ME. Interventions in pain management for persons with an intellectual disability. J Intellect Disabil. 2019;23(1):1-13.

14. Hauer J, Houtrow AJ. Pain assessment and treatment in children with significant impairment of the central nervous system. Pediatrics. 2017;139(6):e20171002.

15. Genik LM, McMurtry CM, Breau LM, Lewis SP, Freedman-Kalchman T. Pain in children with developmental disabilities: development and preliminary effectiveness of a pain training workshop for respite workers. Clin J Pain. 2017;34(5):428-37.

16. Parkinson KN, Gibson L, Dickinson HO, Colver A. Pain in children with cerebral palsy: a crosssectional multicentre European study. Acta Paediatr. 2010;99(3):446-51.

17. Burkitt CC, Breau LM, Zabalia M. Parental assessment of pain coping in individuals with intellectual and developmental disabilities. Res Dev Disabil. 2011;32(5):1564-71.

18. Kankkunen $P$, Jänis $P$, Vehviläinen-Julkunen K. Pain assessment among non-communicating intellectually disabled people described by nursing staff. Open Nurs J. 2013;4:55-9.

19. Ely E, Chen-Lim ML, Zarnowsky C, Green R, Shaffer S, Holtzer B. Finding the evidence to change practice for assessing pain in children who are cognitively impaired. J Pediatr Nurs. 2012;27(4):402-10.

20. Quinn BL, Seibold E, Hayman L. Pain assessment in children with special needs: a review of the literature. Except Child. 2015;8(1):44-57.

21. Massaro M, Ronfani L, Ferrara G, Badina L, Giorgi R, D'Osualdo F, et al. A comparison of three scales for measuring pain in children with cognitive impairment. Acta Paediatr Int J Paediatr. 2014;103(11): e495-500.

22. Massaro M, Pastore S, Ventura A, Barbi E. Pain in cognitively impaired children: a focus for general pediatricians. Eur J Pediatr. 2013;172(1):9-14.

23. Quinn BL, Solodiuk JC, Morrill D, Mauskar S. Pain in nonverbal children with medical complexity: a two-year retrospective study. Am J Nurs. 2018;118(8):28-37.

24. Solodiuk JC, Scott-Sutherland J, Meyers M, Myette B, Shusterman C, Karian VE, et al. Validation of the Individualized Numeric Rating Scale (INRS): a pain assessment tool for nonverbal children with intellectual disability. Pain. 2010;150(2):231-6.

25. Chen-Lim ML, Zarnowsky C, Green R, Shaffer S, Holtzer B, Ely E. Optimizing the assessment of pain in children who are cognitively impaired through the quality improvement process. J Pediatr Nurs. 2012;27(6):750-9.

26. Valkenburg AJ, Leeuw TG, Dijk M, Tibboel D. Pain in intellectually disabled children: towards evidence-based pharmacotherapy? Pediatr Drugs. 2015;17:339-48.

27. Carter B,Arnott J, Simons J, Bray L. Developing a sense of knowing and acquiring the skills to manage pain in children with profound cognitive impairments: mothers' perspectives. Pain Res Manag. 2017;2017:2514920

28. Hadden KL, LeFort S, O'brien M, Coyte PC, Guerreiro DN. A comparison of observers and selfreport pain ratings for children with cerebral palsy. J Dev Behav Pediatr. 2015;36(1):14-23.

29. Barney CC, Stibb SM, Merbler AM, Summers RL, Deshpande S, Krach LE, et al. Psychometric properties of the brief pain inventory modified for proxy report of pain interference in children with cerebral palsy with and without cognitive impairment. Pain Reports. 2018;3(4):e666. 